\title{
Fatores associados à cesariana eletiva em mulheres atendidas em um hospital referência do oeste catarinense
}

Factors associated with elective cesarean section in women met in a reference hospital in western Santa Catarina

Factores asociados con la cesárea electiva en mujeres atendidas en un hospital de referencia del oeste de Santa Catarina

\section{Maíra Rossetto ${ }^{\mathrm{I}}$, Joice Moreira Schmalfuss ${ }^{\mathrm{II}}$, Karine Bedin ${ }^{\mathrm{III}}$, Adelaine Marília Pinheiro ${ }^{\mathrm{IV}}$, Joanna d'Arc Lyra Batista ${ }^{\mathrm{v}}$}

Resumo: Objetivo: identificar os fatores associados à cesariana eletiva em mulheres atendidas em um hospital referência do oeste catarinense. Método: estudo transversal realizado com mulheres submetidas à cesariana. A coleta de dados ocorreu entre agosto e setembro de 2016, por meio de questionário padronizado e informações complementadas pelo prontuário e cartão pré-natal. Dados foram submetidos à regressão logística multivariada. Resultados: foram incluídas no estudo 206 mulheres. O resultado final do modelo multivariado mostrou associação de forma independente com a cesariana eletiva ter sido paga via particular ou por plano de saúde ( Odds Ratio $=39,9$ $\mathrm{p}<0,01)$ e a cor da pele autorreferida branca (Odds Ratio $=2,94 \mathrm{p}=0,035)$. Conclusão: é necessário repensar a formação dos profissionais da área da saúde habilitados a atenderem gestantes, capacitando-os para o cuidado prénatal e para o parto vaginal, visando, sobretudo, o respeito às indicações absolutas de cesarianas, especialmente nas gestantes atendidas no setor privado.

Descritores: Saúde da Mulher; Tocologia; Cesárea; Fatores de Risco; Saúde Suplementar

Abstract: Objective: to identify factors associated with elective cesarean section in women met in a reference hospital in western Santa Catarina. Method: cross-sectional study carried out with women submitted to cesarean section. The data collection occurred between August and September 2016, through a standardized questionnaire and information complemented by the medical records and prenatal card. Data were submitted to multivariate logistic regression. Results: 206 women were included in the study. The final result of the multivariate model

\footnotetext{
${ }^{\text {I }}$ Enfermeira. Doutora. Professora do Curso de Graduação em Medicina da Universidade Federal da Fronteira Sul (UFFS), Chapecó, Santa Catarina, Brasil. E-mail: maira.rossetto@uffs.edu.br ORCID: https://orcid.org/0000-0002-5683-4835

II Enfermeira. Doutoranda. Professora do Curso de Graduação em Enfermagem da Universidade Federal da Fronteira Sul (UFFS), Chapecó, Santa Catarina, Brasil. E-mail: joice.schmalfuss@uffs.edu.br ORCID: https://orcid.org/0000-0002-0293-9957

III Acadêmica do Curso de Graduação em Medicina da Universidade Federal da Fronteira Sul (UFFS), Chapecó, Santa Catarina, Brasil. E-mail: karine.bedin@gmail.com ORCID: https://orcid.org/0000-0001-7437-8186

IV Acadêmica do Curso de Graduação em Medicina da Universidade Federal da Fronteira Sul (UFFS), Chapecó, Santa Catarina, Brasil. E-mail: adelainepinheiro@gmail.com ORCID: https://orcid.org/0000-0001-8722-8478

v Biomédica. Doutora. Professora do Curso de Graduação em Medicina da Universidade Federal da Fronteira Sul (UFFS), Chapecó, Santa Catarina, Brasil. E-mail: joanna.batista@uffs.edu.br ORCID: https://orcid.org/0000-0002-3703-2845
} 
Fatores associados à cesariana eletiva em mulheres atendidas em um hospital refere... | 2

showed independent association with the elective cesarean section paid privately or by health insurance (Odds Ratio $=39.9 \mathrm{p}<0.01$ ) and self-reported skin color white (Odds Ratio $=2.94 \mathrm{p}=0.035$ ). Conclusion: it is necessary to rethink the training of health care professionals qualified to meet pregnant women, empowering them to prenatal care and to the vaginal delivery, aiming, above all, to respect absolute indications of cesarean sections, especially in pregnant women met in the private sector.

Descriptors: Women's Health; Midwifery; Cesarean Section; Risk Factors; Supplementary Health

Resumen: Objetivo: identificar los factores asociados con la cesárea electiva en mujeres atendidas en un hospital de referencia del oeste de Santa Catarina. Método: estudio transversal realizado con mujeres sometidas a cesárea. La recogida de datos se produjo entre agosto y septiembre de 2016, por medio de un cuestionario estandarizado y complementada por la información de los registros médicos y cartón prenatal. Los datos obtenidos fueron sometidos a la regresión logística multivariada. Resultados: se incluyeron 206 mujeres en el estudio. El resultado final del modelo multivariado mostró asociación independiente con la cesárea electiva ha sido pagada en privado o por un plan de salud (Odds Ratio $=39,9 \mathrm{p}<0,01$ ) y autorreporte del color de la piel blanco (Odds Ratio $=2,94 \mathrm{p}=0,035$ ). Conclusión: es necesario repensar la formación de los profesionales de atención de salud calificados para atender a las mujeres embarazadas, motivándolos a la atención prenatal y al parto vaginal, destinado, sobre todo, al respeto de las indicaciones absolutas de cesárea, especialmente en embarazadas atendidas en el sector privado.

Descriptores: Salud de la Mujer; Partería; Cesárea; Factores de Riesgo; Salud Suplemental

\section{Introdução}

A assistência ao parto tem sido marcada por intervenções tecnológicas e ampla utilização da cesariana como forma de nascer. Apesar das recomendações da Organização Mundial da Saúde (OMS), do Ministério da Saúde (MS), de associações, federações e conselhos federais e regionais de Medicina e de Enfermagem relacionadas à obstetrícia, de programas e políticas públicas de saúde, bem como de redes e organizações da sociedade civil, o Brasil segue sendo um dos países do mundo que mais utiliza a técnica cirúrgica para o nascimento. ${ }^{1}$

Culturalmente, a cesárea deixou de ser um procedimento reservado às gestações de alto risco e passou a ser um procedimento agendado, muitas vezes, sem necessidade real de indicação. Um reflexo disso se expressa por índices crescentes de cesáreas no Brasil, que ultrapassaram o parto vaginal, pela primeira vez, em $2009 .^{2}$

Os últimos dados disponíveis sobre nascidos vivos no Departamento de Informática do Sistema Único de Saúde (DATASUS)² mostram que, em 2017, o Brasil contabilizou um total de 2.923.535 nascimentos. Destes, 1.627 .302 ocorreram por cesariana e 1.294 .034 via vaginal, 
3 | Rossetto M, Schmalfuss JM, Bedin K, Pinheiro AM, Batista JDL

demonstrando que os índices desse tipo de cirurgia continuam ultrapassando os de parto. $\mathrm{Na}$ região Sul ocorreram 397.323 nascimentos, sendo que 243.780 (61,3\%) foram por via alta. Já em Santa Catarina, do total de 98.279 nascimentos, 57.012 (58\%) ocorreram por meio de uma operação cesárea. ${ }^{3}$

Essa modificação na forma de nascer dos brasileiros tem tornado as cesarianas a primeira opção para muitas mulheres, perfazendo $56,7 \%$ de todos os nascimentos ocorridos no país, sendo que este percentual chega a $85 \%$ nos serviços privados de saúde. ${ }^{4}$ Nesse âmbito, importante declaração da OMS alerta que, ao nível populacional, taxas de cesárea maiores que $10 \%$ não estão associadas com a redução da mortalidade materna e neonatal. ${ }^{5}$

Dessa forma, por mais que a cesariana seja considerada uma cirurgia segura e importante para a saúde materna e infantil, ${ }^{4}$ quando realizada de maneira indiscriminada e não fundamentada em evidências científicas, pode expor as mulheres e seus recém-nascidos a riscos desnecessários. ${ }^{6}$

Nesse contexto, um estudo evidenciou que o risco de morte materna pós-cesárea foi quase três vezes maior quando comparado ao pós-parto vaginal em decorrência de hemorragia

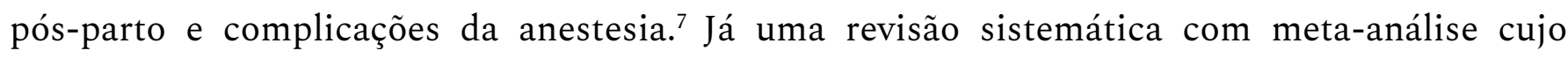
objetivo foi determinar os riscos de complicações maternas agudas graves associadas à cesariana sem indicação médica corroborou com as maiores chances de ocorrência de morte materna após essa cirurgia e também verificou maiores chances de infecção pós-parto. ${ }^{8}$

Dados do SUS também mostraram maiores riscos de infecção puerperal (4,35 vezes mais) e de mortalidade materna (3 vezes mais) em mulheres submetidas à cesárea quando comparadas a mulheres que tiveram parto vaginal ou abortamento. ${ }^{9}$ Outros riscos como lesão acidental de órgãos e infecção de sítio cirúrgico também foram observados. ${ }^{9}$ Importante ressaltar que risco de infecções e complicações cirúrgicas aumentam em locais que não possuem infraestrutura e/ou capacidade para a realização de cesarianas de maneira segura. ${ }^{5}$ 
Fatores associados à cesariana eletiva em mulheres atendidas em um hospital refere... $\mid 4$

Mediante o exposto e considerando que existem lacunas de conhecimento científico produzido em relação às vias de nascimento na região oeste de Santa Catarina, aliado aos altos índices de cesariana nesse Estado, torna-se importante desenvolver pesquisas sobre essa problemática visando compreender a situação, suas causas e consequências no intuito de implementar ações que possam reduzir os altos índices atualmente observados. Nesse sentido, a presente pesquisa objetivou identificar os fatores associados à cesariana eletiva em mulheres atendidas em um hospital referência do oeste catarinense.

\section{Método}

Foi realizado um estudo base transversal com todas as puérperas internadas na maternidade de um hospital referência do oeste catarinense, independente de terem sido submetidas a parto vaginal ou à cesariana. Foram critérios de inclusão: ser puérpera, estar nas primeiras 48 horas de pós-parto/pós-cesárea e estar internada na maternidade do referido hospital. Os critérios de exclusão foram: ter idade inferior a 18 anos, não falar português e estar em condições clínicas que impossibilitaram a participação no estudo (exemplo: cefaleia pósanestesia, dor pela cirurgia cesariana). Ressalta-se que se estabeleceu um critério de exclusão relacionado ao idioma em virtude do oeste catarinense ter recebido, nos últimos anos, muitos imigrantes haitianos e estes apresentarem dificuldades de compreensão e de fala da língua portuguesa.

A coleta de dados foi realizada entre agosto e setembro de 2016, por meio de questionário padronizado aplicado pelas pesquisadoras na beira do leito de cada puérpera. O questionário foi elaborado especialmente para essa pesquisa e abordou informações referentes aos antecedentes clínicos e obstétricos, ao parto atual, ao(s) recém-nascido(s) e ao pré-natal. Informações foram complementadas com consulta ao prontuário e ao cartão pré-natal de cada puérpera, incluindo número de gestações, de partos, de cesarianas e de abortos, número de consultas pré-natais, 
5 | Rossetto M, Schmalfuss JM, Bedin K, Pinheiro AM, Batista JDL

idade gestacional de acordo com a primeira ecografia e com a data da última menstruação, resultados de exames, motivo ou indicação da cesariana, apgar do recém-nascido, entre outras. A fim de evitar viés de seleção, as participantes incluídas na pesquisa foram selecionadas por meio de plantão das pesquisadoras, nos sete dias da semana, ao passo que todas as puérperas internadas na maternidade do hospital do estudo foram convidadas a participar.

O hospital onde a pesquisa foi desenvolvida é responsável por atender cerca de 92 municípios da região oeste de Santa Catarina e 26 municípios do Paraná e Rio Grande do Sul, cobrindo aproximadamente uma população de 1.000.000 de habitantes. Dessa forma, tal instituição de saúde é referência de atendimento para mulheres em trabalho de parto, parto e pós-parto, oferecendo serviços pelo sistema público de saúde, por convênios de saúde e de forma particular.

No presente estudo analisaram-se apenas as cesarianas dentre a amostra total do estudo base. A variável dependente foi cesariana eletiva cujo registro no prontuário fosse "parto por cesariana eletiva" (CID-10 O82.0) e/ou cesariana relatada pela puérpera no momento da aplicação do questionário. As variáveis independentes foram analisadas como associadas à cesárea e divididas em blocos de variáveis: biológicas, socioeconômicas, clínicas, relacionadas ao serviço de saúde e ao recém-nascido.

Dados coletados no DATASUS mostraram que Chapecó contabilizou 3.183 nascidos vivos em 2013 (ano em que o projeto foi construído), sendo que 74 nascimentos foram decorrentes de gestação dupla, nenhuma de gestação tripla e três de tipo de gestação ignorada. Considerando-se estes dados, levantou-se o número de 3.146 puérperas no ano de 2013. A partir desses achados e de percentuais de cesariana descritos na literatura, ${ }^{10}$ foi calculado o tamanho de amostra de 156 puérperas, considerando um erro amostral de 5\% e um nível de confiança de 95\%.

As análises de fatores associados foram feitas por meio de regressão logística multivariada, com emprego do Odds Ratio. A regressão logística permite calcular ou prever a 
Fatores associados à cesariana eletiva em mulheres atendidas em um hospital refere... $\mid 6$

probabilidade de um evento específico (variável dependente) ser explicado por meio de múltiplas variáveis independentes. Após análise bivariada, ou seja, da variável dependente com cada uma das variáveis independentes, as variáveis independentes que obtiveram um $\mathrm{p}$ valor $<0,20$ foram acrescentadas no modelo multivariado forward por blocos de variáveis, permanecendo no modelo final apenas as variáveis com associação $\mathrm{p}<0,05$. No modelo multivariado forward as variáveis são acrescentadas uma a uma de acordo com sua força de associação (do menor para o maior p valor). Os dados foram analisados por meio do programa Stata versão 12.

O estudo seguiu os preceitos éticos da Resolução do Conselho Nacional de Saúde número 466, de 2012 e foi aprovado pelo Comitê de Ética em Pesquisa da Universidade Federal da Fronteira Sul, sob parecer de número 1.575.071, em 5 de junho de 2016. Todas as participantes assinaram o Termo de Consentimento Livre e Esclarecido antes de responderem ao questionário, bem como preliminarmente ao acesso de informações dos seus prontuários e/ou cartão pré-natal.

\section{Resultados}

Das puérperas que se adequaram aos critérios de inclusão, três recusaram participar do estudo, totalizando a participação de 343 mulheres. Destas, 206 (60,1\%) foram submetidas à cirurgia cesariana e compuseram a amostra.

As características das puérperas incluídas estão descritas na Tabela 1. 
Tabela 1- Características das cesarianas realizadas em hospital de referência do Oeste Catarinense. Chapecó-SC, 2016.

\begin{tabular}{|c|c|c|}
\hline Características & Total n (\%) & Média (mín. - máx.) \\
\hline \multicolumn{3}{|l|}{ Sociodemográficas } \\
\hline Idade & & $28,8(18-42)$ \\
\hline \multicolumn{3}{|l|}{ Cor da pele autorreferida } \\
\hline Branca & $126(61,2)$ & \\
\hline Parda ou morena & $67(32,5)$ & \\
\hline Preta & $05(2,4)$ & \\
\hline Amarela ou oriental & $02(1,0)$ & \\
\hline Indígena & $04(1,9)$ & \\
\hline Não sabe & $02(8,0)$ & \\
\hline \multicolumn{3}{|l|}{ Variáveis clínicas e laboratoriais } \\
\hline \multicolumn{3}{|l|}{ Eventos de risco durante gestação } \\
\hline Diabetes gestacional & $12(5,8)$ & \\
\hline Pressão alta & $32(15,5)$ & \\
\hline Placenta prévia & $07(0,34)$ & \\
\hline Eclâmpsia ou convulsão & $09(4,4)$ & \\
\hline Cerclagem & $03(1,5)$ & \\
\hline Ruptura uterina & $01(0,5)$ & \\
\hline Idade gestacional média (em semanas) & & $38,6(28-42)$ \\
\hline Pré-termo (menor que 37 semanas de gestação) & $21(10,2)$ & \\
\hline Termo (entre 37 e 41 semanas e 6 dias) & $182(88,3)$ & \\
\hline Pós-termo (42 semanas ou mais) & $03(1,5)$ & \\
\hline Vírus da Imunodeficiência Humana (HIV) reagente & $00(0,0)$ & \\
\hline Sifilis (VDRL) reagente & $01(0,5)$ & \\
\hline \multicolumn{3}{|l|}{ Variáveis relacionadas ao serviço de saúde } \\
\hline Realização de pré-natal & $206(100 \%)$ & \\
\hline \multicolumn{3}{|l|}{ Variáveis relacionadas ao recém-nascido } \\
\hline Peso do recém-nascido & & $3126,9(950-4570)$ \\
\hline Peso do recém-nascido gemelar & & $1952,1(625-2940)$ \\
\hline Apgar do primeiro minuto & & $9(1$ a 10$)$ \\
\hline Apgar do primeiro minuto do gemelar & & $8(8$ a 9$)$ \\
\hline
\end{tabular}

A idade média das participantes foi de 28,8 anos $(18$ - 42), todas realizaram pré-natal e o percentual de partos prematuros foi de 10,2\% (21). As cesáreas eletivas corresponderam a $88,3 \%$ (182) dos nascimentos a termo e a idade gestacional média das participantes, no momento de 
Fatores associados à cesariana eletiva em mulheres atendidas em um hospital refere... $\mid 8$

interrupção da gestação, foi de 38,6 semanas. Os recém-nascidos apresentaram índice de apgar favorável no primeiro e no quinto minuto de vida.

A Tabela 2 apresenta os resultados das análises bivariadas das variáveis avaliadas por cesariana eletiva no hospital onde a pesquisa foi realizada. Foram para a análise multivariada as seguintes variáveis: idade materna de 30 a 42 anos, tipo de pagamento da cesariana privado, ter algum evento durante a gestação e ter realizado três ou mais ultrassonografias durante a gravidez. Optou-se por acrescentar a variável cor da pele autorreferida branca no modelo multivariado por esta apresentar um $\mathrm{p}$ valor boderline.

No presente estudo algumas variáveis como intercorrências durante a gestação, número de consultas pré-natais e cesáreas anteriores não se apresentaram significativas quando relacionadas à cesariana eletiva.

Tabela 2- Frequências e análise bivariada dos fatores biológicos, socioeconômicos, clínicos, relacionados ao serviço de saúde e ao recém-nascido associados à cesariana eletiva. ChapecóSC, 2016.

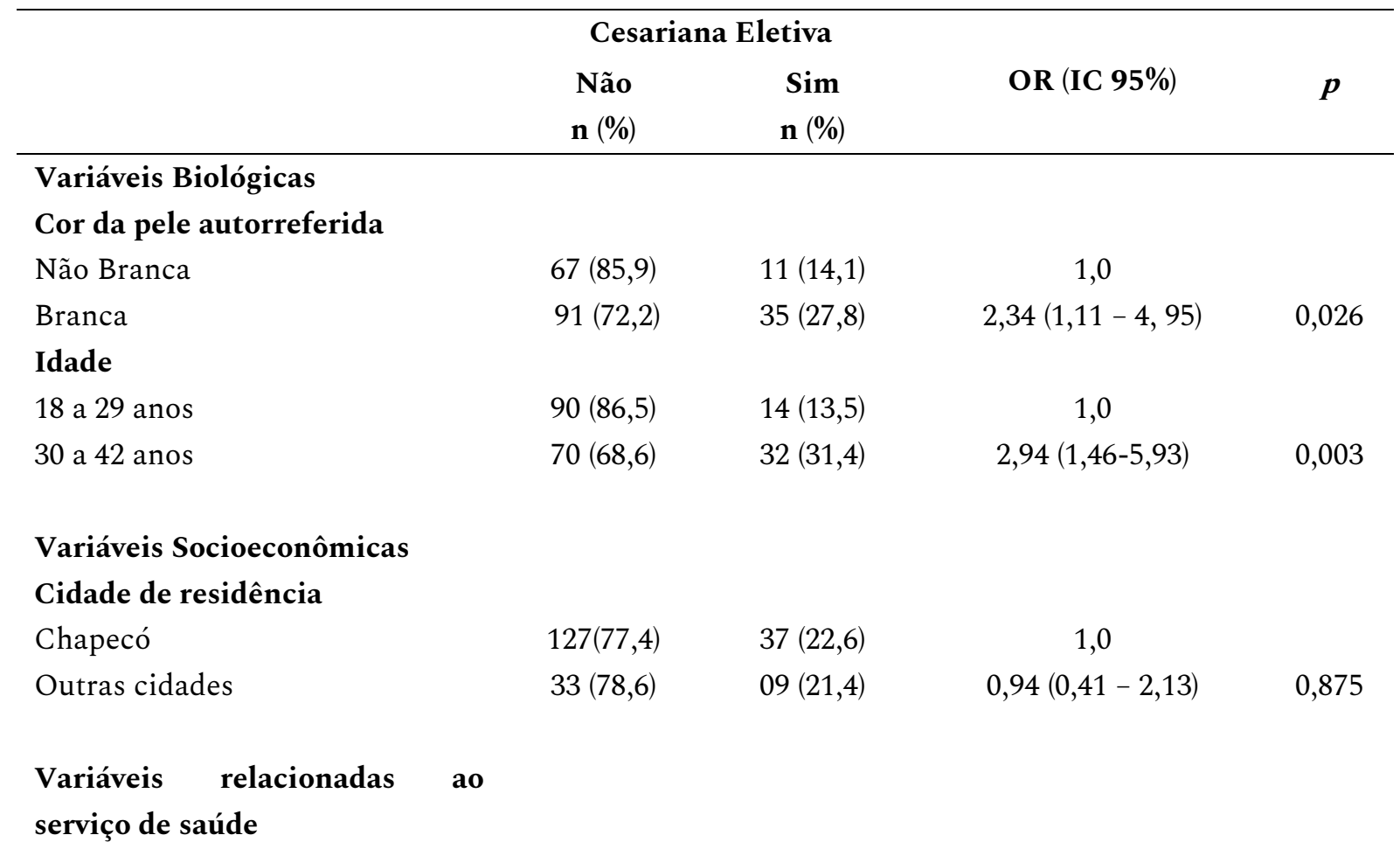


9 | Rossetto M, Schmalfuss JM, Bedin K, Pinheiro AM, Batista JDL

Tipo de pagamento da cesariana

Público

$146(93,6) \quad 10(6,4)$

1,0

Privado

$14(28,0)$

$36(72,0)$

$37,54(15,4-91,4) \quad 0,000$

Acompanhamento do pré-natal

Profissionais diferentes

$49(86,0)$

$8(14,0)$

1,0

Sempre ou maior parte com o

$111(74,5)$

$38(25,5)$

$2,10(0,91-4,82)$

0,082

mesmo profissional

\section{Variáveis clínicas}

Cesariana anterior

Não

$84(77,1)$

$25(23,0)$

1,0

Sim

$76(78,3)$

$21(21,7)$

$0,93(0,48-1,79)$

0,825

Ter algum evento na gravidez atual $^{*}$

Não

$118(73,7)$

$42(26,3)$

1,0

Sim

$42(91,3)$

$4(8,7)$

$0,27(0,09-0,80)$

0,017

Número de consultas no pré-

natal

Seis ou mais

Cinco ou menos

$132(78,1)$

$37(21,9)$

1,0

Número de ultrassonografia(s)

$16(76,2)$

$5(23,8)$

$1,11(0,38-3,24)$

0,842

durante a gravidez

Até duas

$40(90,9)$

$04(9,1)$

1,0

Três ou mais

$116(73,4)$

$42(26,6)$

$3,62(1,22-10,73)$

0,020

Variáveis relacionadas ao

recém-nascido

Bebê diagnosticado com agravo

Não

$148(76,7)$

$45(23,3)$

1,0

Sim

$12(92,3)$

$01(7,70)$

$0,30(0,03-2,16)$

0,220

Peso do recém-nascido

$<3000 \mathrm{~g}$

$56(71,8)$

$22(28,2)$

1,0

$\geq 3000 \mathrm{~g}$

$94(79,7)$

$24(20,3)$

$0,65(0,33-1,26)$

0,205

Apgar do $1^{\circ}$ minuto

7 a 10

$142(76,0) \quad 45(24,0)$

1,0

0 a 6

$12(92,3)$

$01(7,7)$

$0,26(0,03-2,10)$

0,205

* Diabetes gestacional, pressão alta, placenta prévia, eclâmpsia, cerclagem e/ou ruptura uterina.

Foi realizada uma análise multivariada forward por bloco de variável, sendo cada uma acrescentada de acordo com a força de associação e retirada caso não permanecesse significativa. $\mathrm{O}$ resultado do modelo multivariado é apresentado na Tabela 3 , permanecendo 
Fatores associados à cesariana eletiva em mulheres atendidas em um hospital refere... | 10

associadas de forma independente com a cesariana eletiva: ter a cirurgia paga por via particular ou por plano de saúde $(\mathrm{OR}=39,9 \mathrm{p}<0,01)$ e a cor da pele autorreferida branca $(\mathrm{OR}=2,94 \mathrm{p}=0,035)$.

Tabela 3- Modelo final da regressão multivariada forward da associação entre exposições estudadas e cesariana eletiva. Chapecó-SC, 2016.

\begin{tabular}{lcc}
\hline & OR $(\mathbf{I C}$ 95\%) & $\boldsymbol{p}$ \\
\hline $\begin{array}{l}\text { Tipo de pagamento da cesariana } \\
\text { Público }\end{array}$ & 1,0 \\
Privado & $39,9(15,8-101,1)$ & 0,000 \\
& & \\
Cor da pele autorreferida & 1,0 & \\
Não branca & $2,94(1,08-8,03)$ & 0,035 \\
Branca &
\end{tabular}

\section{Discussão}

Os resultados mostraram que todas as mulheres submetidas à cesariana eletiva tiveram acompanhamento pré-natal, com realização de ultrassonografias e com poucos eventos de risco durante a gravidez, tais como: diabetes gestacional, pressão alta, placenta prévia, eclâmpsia, cerclagem e/ou ruptura uterina. Mesmo se tratando de um hospital referência para toda a região oeste do Estado de Santa Catarina, a maioria das cesáreas foi de mulheres residentes em Chapecó. Ter a cirurgia de forma privada (particular ou por meio de plano de saúde) e ter a cor da pele autorreferida como branca ficaram associadas à realização da cesárea eletiva.

Um estudo brasileiro apontou associação entre a cesárea em mulheres com nível socioeconômico elevado e que utilizaram o setor privado. Além disso, a alta escolaridade foi associada como um maior risco para a realização de cesárea eletiva. ${ }^{11}$

O tipo de pagamento privado esteve fortemente associado à cesariana eletiva, apresentando uma chance 40 vezes maior quando comparado às mulheres que tiveram parto pelo sistema público de saúde. Outros estudos também evidenciaram uma maior ocorrência de 
cesarianas no setor privado em comparação com o setor público. Das mulheres que tiveram o desfecho gestacional pelo sistema privado, 93,8\% ocorreu por meio de cesariana, e das que tiveram parto pelo SUS, 55,5\% ocorreu pela via de nascimento abdominal. ${ }^{12-13}$ Pesquisa apontou uma taxa de cesariana de $51,9 \%$, sendo $42,9 \%$ no setor público e $87,9 \%$ no setor privado ${ }^{10}$ e outro estudo verificou que nas internações que ocorreram via particular ou por convênio de saúde, a cesárea foi 2,43 vezes mais prevalente quando comparada à internação pelo SUS. ${ }^{6}$

Desta forma, a ocorrência de cesariana eletiva pode estar associada a um maior poder aquisitivo que, muitas vezes, facilita o pagamento por este serviço, visto que, em muitos casos, deixou de ser um método para melhorar os resultados perinatais e tornou-se um produto de consumo, de tal maneira que suas taxas são menores entre as mulheres mais pobres, aumentando na proporção em que aumenta o poder aquisitivo da população. ${ }^{12}$ Um estudo encontrou relação entre a preferência pela via alta de nascimento e melhor condição socioeconômica (escolaridade e maior nível econômico). ${ }^{14}$

Mulheres do setor privado apresentaram $87,5 \%$ de cesárea, com aumento da decisão pela via abdominal de nascimento no final da gestação, independentemente do diagnóstico de complicações. $^{2}$ O desestímulo ao parto vaginal foi fortemente observado durante o pré-natal realizado pelo sistema privado, em que o acompanhamento é exclusivo com o médico, enquanto no SUS, em muitos municípios, as consultas são alternadas entre profissionais da Medicina e da Enfermagem..$^{12}$ Outro estudo também apontou que a escolha da cesariana sofre forte influência da atuação médica. ${ }^{14}$

Pesquisa transversal que objetivou identificar fatores sociodemográficos, características e intercorrências gestacionais associadas à realização de cesárea eletiva corrobora com as evidências recém expostas. ${ }^{15} \mathrm{Nela}$, as gestantes acompanhadas na rede de saúde suplementar apresentaram maior chance de cesárea eletiva, sendo comprovada a associação entre essa modalidade de nascimento e local de pré-natal e parto. ${ }^{15}$ 
Fatores associados à cesariana eletiva em mulheres atendidas em um hospital refere... | 12

A cesariana marcada com antecedência pode ser percebida como um novo tipo de nascimento em que se elimina a imprevisibilidade, além de permitir o planejamento e a participação de familiares e amigos no parto e no período pós-parto. Esses aspectos de ordem social também se relacionam ao aumento crescente dessa via de nascimento. ${ }^{11}$

Uma investigação que utilizou dados da pesquisa Nascer no Brasil atribuiu o agendamento prévio de cesáreas a uma “contaminação” causada pela maneira como os hospitais privados se organizam, considerando tanto o desejo da gestante quanto a comodidade do profissional médico. ${ }^{16}$

O predomínio de cesarianas eletivas também pode estar relacionada a uma falsa percepção de que esta cirurgia é um modo de nascimento mais seguro, menos dolorido e símbolo de status social para muitas mulheres brasileiras. ${ }^{11}$ Nesse sentido, uma pesquisa verificou que entre as mulheres do setor privado houve maior percepção de que a cesariana é a via de nascimento mais segura para o bebê quando comparada à realização do parto vaginal. ${ }^{2}$ Assim, a escolha pela cesárea pode ser influenciada por diversos motivos, estando relacionada às decisões da própria mulher, dos profissionais e serviços da saúde e, inclusive, por pressões familiares e da sociedade.

$\mathrm{Na}$ presente pesquisa, ter a cor da pele branca esteve associada três vezes mais à realização de cesariana eletiva do que outras cores de pele, mesmo com o controle pelo tipo de pagamento. Um estudo também demonstrou maior proporção dessa via de nascimento entre mulheres brancas. ${ }^{17}$

Investigação realizada na Califórnia demonstrou que mulheres de descendência afroamericana apresentaram taxas mais elevadas de cesáreas quando comparadas com outras mulheres. ${ }^{18}$ Também ressaltou que a má comunicação entre profissionais da saúde e mulheres pode ser um fator de risco importante para a alta taxa de cesáreas realizadas. No entanto, nos resultados encontrados, mulheres afro-americanas que tiveram cesáreas anteriores não 
apresentaram uma probabilidade significativa de realizar uma nova cesárea, seja ela eletiva ou de emergência. ${ }^{18}$

Em contrapartida, uma pesquisa brasileira relacionou a cor da pele branca a maiores taxas de cesárea, principalmente quando a cirurgia ocorreu pelo sistema privado. Além disso, mostrou que mulheres que tiveram uma cesárea prévia apresentaram maiores chances de serem submetidas a outra cesárea, quando se comparou a via de parto prévia como sendo vaginal. ${ }^{19}$

Mulheres de cor branca, quando comparadas a mulheres de cor preta e parda, apresentaram maior risco de terem um pré-natal inadequado, falta de vinculação à maternidade, ausência de acompanhante, peregrinação para o parto e menos anestesia local para episiotomia. ${ }^{20}$ Desta forma, foram identificadas diferenças na assistência evidenciadas por disparidades raciais no processo de atenção à gestação e ao parto, com gradiente de pior para melhor cuidado entre mulheres pretas, pardas e brancas, respectivamente.

Diante do exposto, torna-se importante mencionar que o hospital que serviu como local de estudo para a coleta de dados da presente pesquisa ainda não adota a Classificação de Robson, ${ }^{5}$ conforme sugerido pela OMS. Tal sistema de classificação considera cinco características obstétricas, categorizando as gestantes em um dos 10 grupos existentes. ${ }^{5}$ Somase a não utilização desse instrumento o fato de que as condutas dos obstetras, muitas vezes, são pautadas nas escolhas do médico plantonista do dia, visto que protocolos ainda estão sendo construídos no que tange a algumas situações específicas do atendimento obstétrico.

\section{Conclusão}

Ter a cesariana de forma privada (particular ou por meio de plano de saúde) e ter a cor da pele autorreferida como branca ficaram associadas à realização da cesárea eletiva no resultado final do modelo multivariado, sugerindo que mulheres que dispõem de melhores condições econômicas podem optar por esse procedimento cirúrgico com maior facilidade. A cor da pele 
Fatores associados à cesariana eletiva em mulheres atendidas em um hospital refere... | 14

autorreferida branca também pode demonstrar que a assistência ao parto das mulheres depende da classe socioeconômica, já que mulheres de cor de pele parda ou negra são mais submetidas ao parto vaginal do que à cesariana eletiva.

Assim, torna-se necessário repensar a formação dos profissionais da área da saúde habilitados a atenderem gestantes, capacitando-os para o cuidado pré-natal e para o parto vaginal, visando, sobretudo, o respeito às indicações absolutas de cesarianas, especialmente nas gestantes atendidas no setor privado. Por isso, acredita-se no potencial desse manuscrito para contribuir cientificamente nas atividades relacionadas ao ensino, para problematizar e fomentar futuros projetos de pesquisa e de extensão e para qualificar a assistência.

Uma vez que o presente estudo teve caráter quantitativo, recomenda-se que outras investigações avaliem de forma qualitativa o processo de cesárea eletiva por meio de uma abordagem que considere o desejo da mulher quanto à via de parto, com posterior satisfação quanto a sua escolha. Outra possibilidade seria a análise a respeito da visão da equipe médica sobre os riscos e benefícios da cesárea eletiva.

\section{Referências}

1. Entringer AP, Pinto M, Dias MAB, Gomes MASM. Análise de custo-efetividade do parto vaginal espontâneo e da cesariana eletiva para gestantes de risco habitual no Sistema Único de Saúde. Cad Saúde Pública. 2018;34(5):e00022517. doi: http://dx.doi.org/10.1590/0102-311X00022517

2. Domingues RMSM, Dias MAB, Nakamura-Pereira M, Torres JA, d'Orsi E, Pereira APE, et al. Processo de decisão pelo tipo de parto no Brasil: da preferência inicial das mulheres à via de parto final. Cad Saúde Pública. 2014;30 Supl 1:101-16. doi: http://dx.doi.org/10.1590/0102-311X00105113

3. Ministério da Saúde (BR). Departamento de Informática do Sistema Único de Saúde (DATASUS). Sistema de Informações sobre Nascidos Vivos (SINASC). Brasília (DF): Ministério da Saúde; 2020 [acesso em 2020 mar 29]. Disponível em: http://www2.datasus.gov.br/DATASUS/index.php?area=060702

4. Ministério da Saúde (BR). Secretaria de Atenção à Saúde. Diretrizes de Atenção à Gestante: a operação cesariana. Brasília (DF): Ministério da Saúde; 2016 [acesso em 2020 mar 25]. 115 p. Disponível em: http://conitec.gov.br/images/Consultas/Relatorios/2016/Relatorio_Diretrizes_Cesariana_N179.pdf 
5. Organização Mundial da Saúde (OMS). Declaração da OMS sobre taxas de cesáreas [Internet]. Genebra: OMS; 2015 [acesso em 2020 mar 25]. Disponível em: https://apps.who.int/iris/bitstream/handle/10665/161442/WHO_RHR_15.02_por.pdf

6. Saraiva JM, Gouveia HG, Gonçalves AC. Fatores associados a cesáreas em um hospital universitário de alta complexidade do sul do Brasil. Rev Gaúch Enferm. 2017;38(3):e69141. doi: https://doi.org/10.1590/1983-1447.2017.03.69141

7. Esteves-Pereira AP, Deneux-Tharaux C, Nakamura-Pereira M, Saucedo M, Bouvier-Colle MH, Leal MC. Caesarean delivery and postpartum maternal mortality: a population-based case control study in Brazil. PLoS One. 2016;11(4):e0153396. doi: 10.1371/journal.pone.0153396

8. Mascarello KC, Horta BL, Silveira MF. Maternal complications and cesarean section without indication: systematic review and meta-analysis. Rev Saúde Pública. 2017;51:105. doi: https://doi.org/10.11606/S1518-8787.2017051000389

9. Agência Nacional de Vigilância Sanitária (BR), Gerência de Vigilância e Monitoramento em Serviços de Saúde (GVIMS), Gerência Geral de Tecnologia em Serviços de Saúde (GGTES). Medidas de prevenção e critérios diagnósticos de infecções puerperais em parto vaginal e cirurgia cesariana. Brasília (DF): Anvisa; 2017 [acesso em 2020 mar 25]. Disponível em: http://portal.anvisa.gov.br/documents/33852/3507912/Caderno+8+-

+ Medidas+de+Preven\%C3\%A7\%C3\%A3o+e+Crit\%C3\%A 9rios+Diagn\%C3\%B3sticos+de+Infec\%C3\%A7\%C $3 \% \mathrm{~B} 5 \mathrm{es}+$ Puerperais+em+Parto+Vaginal+e+Cirurgia + Cesariana/08dee73e-ffef-433f-8fb8-c5f7fc8053a0

10. Nakamura-Pereira M, Leal MC, Esteves-Pereira AP, Domingues RMSM, Torres JA, Dias MAB, et al. Use of Robson classification to asses cesarean section rate in Brazil: the role of source of payment for childbirth. Reprod Health. 2016;13(Suppl 3):128. doi: https://doi.org/10.1186/s12978-016-0228-7

11. Riscado LC, Jannotti CB, Barbosa RHS. A decisão pela via de parto no Brasil: temas e tendências na produção da saúde coletiva. Texto \& Contexto Enferm. 2016;25(1):e3570014. doi: http://dx.doi.org/10.1590/0104-0707201600003570014

12. Oliveira RR, Melo EC, Novaes ES, Ferracioli PLRV, Mathias TAF. Fatores associados ao parto cesárea nos sistemas público e privado de atenção à saúde. Rev Esc Enferm USP. 2016;50(5):734-41. doi: http://dx.doi.org/10.1590/S0080-623420160000600004

13. Leal MC, Esteves-Pereira AP, Nakamura-Pereira M, Torres JA, Domingues RMSM, Dias MAB, et al. Provider-initiated late preterm births in Brazil: differences between public and private health services. PLoS One. 2016;11(5):1-14. doi: https://doi.org/10.1371/journal.pone.015551

14. Copelli FHS, Rocha L, Zampieri MFM, Gregório VRP, Custódio ZAO. Fatores determinantes para a preferência da mulher pela cesariana. Texto \& Contexto Enferm. 2015;24(2):336-43. doi: http://dx.doi.org/10.1590/0104-07072015000430014 
Fatores associados à cesariana eletiva em mulheres atendidas em um hospital refere... | 16

15. Ferrari AP, Carvalhaes MABL, Parada CMGL. Associação entre pré -natal e parto na rede de saúde suplementar e cesárea eletiva. Rev Bras Epidemiol. 2016;19(1):75-88. Disponível em: https://doi.org/10.1590/1980-5497201600010007

16. Zaiden L, Nakamura-Pereira M, Gomes MAM, Esteves-Pereira AP, Leal MC. Influência das características hospitalares na realização de cesárea eletiva na Região Sudeste do Brasil. Cad Saúde Pública. 2020;36(1):e00218218. Disponível em: https://doi.org/10.1590/0102-311X00218218

17. Madeiro A, Rufino AC, Santos AO. Partos cesáreos no Piauí: tendência e fatores associados no período 2000-2011. Epidemiol Serv Saúde. 2017;26(1):81-90. doi: http://dx.doi.org/10.5123/s167949742017000100009

18. Huesch M, Doctor JN. Factors associated with increased cesarean risk among African American women: evidence from California, 2010. Am J Public Health. 2015;105(5):956-62. doi: https://doi.org/10.2105/AJPH.2014.302381

19. Freitas PF, Fernandes TMB. Associação entre fatores institucionais, perfil da assistência ao parto e as taxas de cesariana em Santa Catarina. Rev Bras Epidemiol. 2016;19(3):525-38. doi: https://doi.org/10.1590/1980-5497201600030005

20. Leal MC, Gama SGN, Pereira APE, Pacheco VE, Carmo CN, Santos RV. A cor da dor: iniquidades raciais na atenção pré-natal e ao parto no Brasil. Cad Saúde Pública. 2017;33 Supl 1:e00078816. doi: https://doi.org/10.1590/0102-311X00078816

\section{Autora correspondente}

Maíra Rossetto

E-mail: maira.rossetto@uffs.edu.br

Endereço: Universidade Federal da Fronteira Sul (UFFS) - Campus Chapecó

Rodovia SC 484 - Km 02 - Fronteira Sul

Bloco dos Professores - Sala 317

Chapecó, SC, Brasil

CEP: 89815-899

\section{Contribuições de Autoria}

\section{1 - Maíra Rossetto}

Concepção e planejamento do projeto de pesquisa, obtenção ou análise e interpretação dos dados, redação e revisão crítica.

\section{2 - Joice Moreira Schmalfuss}

Concepção e planejamento do projeto de pesquisa, obtenção ou análise e interpretação dos dados, redação e revisão crítica. 
17 | Rossetto M, Schmalfuss JM, Bedin K, Pinheiro AM, Batista JDL

\section{3 - Karine Bedin}

Obtenção e análise dos dados e redação.

\section{4 - Adelaine Marília Pinheiro}

Obtenção e análise dos dados e redação.

\section{5 - Joanna d'Arc Lyra Batista}

Concepção e planejamento do projeto de pesquisa, obtenção ou análise e interpretação dos dados, redação e revisão crítica.

\section{Como citar este artigo}

Rossetto M, Schmalfuss JM, Bedin K, Pinheiro AM, Batista JDL. Fatores associados à cesariana eletiva em mulheres atendidas em um hospital referência do oeste catarinense. Rev. Enferm. UFSM. 2020 [Acesso em: Anos Mês Dia]; vol.10 e54: 1-17. DOI:https://doi.org/10.5902/2179769239398 